\title{
Accelerated simulation of tunable vibration energy harvesting systems using a linearised state-space technique
}

\author{
Leran Wang, Tom J. Kazmierski, Bashir M. Al-Hashimi, \\ Alex S. Weddell, Geoff V. Merrett and Ivo N. Ayala Garcia \\ School of Electronics and Computer Science \\ University of Southampton, UK \\ \{lw04r,tjk,bmah,asw,gvm,inag07r\}@ecs.soton.ac.uk
}

\begin{abstract}
This paper proposes a linearised state-space technique to accelerate the simulation of tunable vibration energy harvesting systems by at least two orders of magnitude. The paper provides evidence that currently available simulation tools are inadequate for simulating complete energy harvesting systems where prohibitive CPU times are encountered due to disparate time scales. In the proposed technique, the model of a complete mixed-technology energy harvesting system is divided into component blocks whose mechanical and analogue electrical parts are modelled by local state equations and terminal variables while the digital electrical part is modelled as a digital process. Unlike existing simulation tools that use Newton-Raphson method, the proposed technique uses explicit integration such as AdamsBashforth method to solve the state equations of the complete energy harvester model in short simulation time. Experimental measurements of a practical tunable energy harvester have been carried out to validate the proposed technique.
\end{abstract}

\section{INTRODUCTION AND RELATED WORK}

Vibration-based energy harvesters convert ambient mechanical vibrations into electrical energy. Environmental vibrations are usually of a small amplitude, therefore the generated electrical energy is typically insufficient to power the electronic circuitry at the application end. A storage element, such as a supercapacitor or a rechargeable battery, is used in such energy harvesting systems to store the generated energy. The load circuit will only wake up and perform computations when enough energy has been accumulated. Most of the reported microgenerator designs are based on spring-mass system with characteristic resonant frequency. These devices generate maximum power when their resonant frequency matches the frequency of the input ambient vibration and normally have high Q-factor. Therefore, the output power generated by microgenerator drops dramatically when there is a difference between the ambient frequency and the resonant frequency. Tunable microgenerators, which can adjust their own resonant frequency through mechanical or electrical methods to match the input frequency, are more desirable and have attracted research interest [1]. A tunable energy harvesting system typically has five key components (Fig. 1): microgenerator which converts ambient environment

978-3-9810801-7-9/DATE11/(C2011 EDAA energy into electrical energy, power processing circuit which regulates the generated voltage, storage element, actuator used for the tuning mechanism and microcontroller that monitors and controls the tunable energy harvesting system.

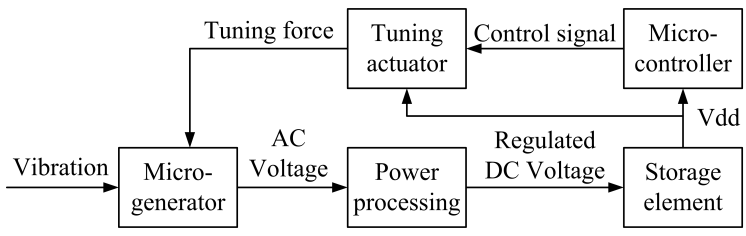

Fig. 1. Block diagram of tunable energy harvesting system

In Fig. 1, the microgenerator and the tuning actuator are mainly mechanical components and the rest of the system are electrical circuits. The most common approach seen in literature is to use Finite Element Modelling (FEM) tools, such as ANSYS and CoventorWare, to simulate the mechanical part. Zhu et al. have developed an electromagnetic microgenerator which is tuned by magnetic force [2]. ANSYS has been used to determine the resonant frequency of the microgenerator cantilever beam and to simulate the magnetic field between the two tuning magnets. Hohlfeld et al. have presented the design of an electrostatic microgenerator which is tuned by electrostatic force [3]. The simulation of the electrostatic field was carried out by CoventorWare. However, the FEM tools cannot be used directly to simulate complete energy harvesting systems because they cannot incorporate the system electrical components. As for the electrical part, SPICE simulators are widely used for circuit simulation and Cadence/SYNOPSYS tools are popular in IC design. There has been reported work on using equivalent circuit models to simulate complete energy harvesting systems. Recently Rao et al. have proposed an energy harvesting simulator based on PSPICE [4]. Wu et al. used equivalent circuit models to simulate their electrical-tuning piezoelectric microgenerator [5]. There are two limitations with the equivalent circuit models approach. Firstly, equivalent circuit models cannot accurately predict the behaviour of a non-tunable energy harvester [6], which implies that this approach will have limitations in modelling tunable energy harvesters. Secondly, circuit simulators take long CPU 
time to simulate complete energy harvesting systems, as we demonstrated in Table I.

A hardware description language (HDL) can model both the mechanical and electrical parts of an energy harvester accurately and efficiently, as presented in [6]. The limitation of the HDL simulation based approach is that available simulators are inadequate for simulating complete, tunable energy harvester systems due to prohibitive CPU times. As an example, we have modelled and simulated the energy harvester reported in [7] using three simulation approaches. The results are shown in Table I. The first simulation is based on a VHDL-AMS model and uses the SystemVision simulator from Mentor Graphics [6]. The second simulation is based on a PSPICE model reported in [4] whilst the third simulation is based on a SystemC-A model [8]. As can be seen in Table I, with each of these simulators a single simulation of supercapacitor charging may take up to 10 hours to finish. Therefore a new technique to accelerate the simulation of energy harvesters is needed, which is the main purpose of this paper.

TABLE I

CPU TIMES OF DIFFERENT SIMULATION ENVIRONMENTS

\begin{tabular}{|c|c|c|c|}
\hline \multicolumn{4}{|c|}{ Simulation of the supercapacitor charging curve of an energy harvester } \\
\hline Simulator & $\begin{array}{c}\text { SystemVision } \\
\text { (VHDL-AMS) }\end{array}$ & $\begin{array}{c}\text { OrCAD } \\
\text { (PSPICE) }\end{array}$ & $\begin{array}{c}\text { Visual C++ } \\
\text { (SystemC-A) }\end{array}$ \\
\hline CPU time (P4, 2G RAM) & 4h 24min & 9h 48min & $6 \mathrm{~h} \mathrm{40min}$ \\
\hline
\end{tabular}

There are two reasons for the long simulation times (Table I). Firstly with reference to Fig. 2, vibration energy harvesters typically have an input frequency of tens or hundreds of hertz in line with the typical environment vibrations encountered, requiring a fine simulation time-step of less than a millisecond. Large supercapacitors used in energy harvesting systems require many hours to fully charge, given the low levels of power generated by typical vibration microgenerators. Secondly, all of the existing HDL simulators use the Newton-Raphson method to solve the energy harvester model's analogue equations at each time step. The Newton-Raphson method is slow in solving such equations [9] leading to long CPU times. We propose to use the a linearised state-space formulation and explicit integration (Adams-Bashforth) to solve the equations in a fast feed-forward process to reduce the simulation time (Section IV).

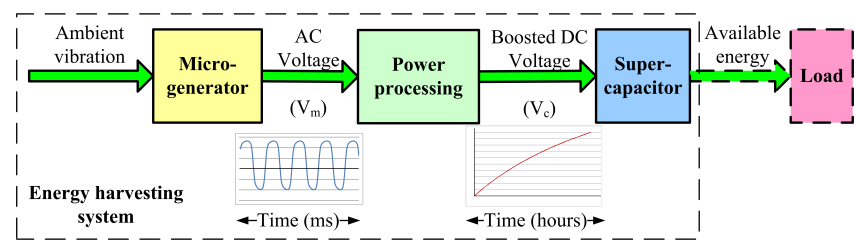

Fig. 2. Power-related components of an energy harvesting system

\section{Proposed LineARISED StATE-SPACE TECHNIQUE}

The analogue part of tunable energy harvester is:

$$
\left[\begin{array}{c}
\dot{\boldsymbol{x}}(t) \\
\mathbf{0}
\end{array}\right]=\left[\begin{array}{l}
\boldsymbol{f}_{x}(\boldsymbol{x}(t), \boldsymbol{y}(t)) \\
\boldsymbol{f}_{y}(\boldsymbol{x}(t), \boldsymbol{y}(t))
\end{array}\right]+\left[\begin{array}{c}
\boldsymbol{e}_{x}(t) \\
\mathbf{0}
\end{array}\right]
$$

where $\boldsymbol{x}: \mathbb{R} \longrightarrow \mathbb{R}^{N}$ is the vector of $N$ state variable waveforms, $\boldsymbol{y}: \mathbb{R} \longrightarrow \mathbb{R}^{M}$ are $M$ non-state variable waveforms, the non-linear functions $\boldsymbol{f}_{x}: \mathbb{R}^{N} \times \mathbb{R}^{M} \longrightarrow \mathbb{R}^{N}$ and $\boldsymbol{f}_{y}: \mathbb{R}^{N} \times \mathbb{R}^{M} \longrightarrow \mathbb{R}^{N}$ represent the relationships between the variables of the model and $e_{x}: \mathbb{R} \longrightarrow \mathbb{R}^{N}$ is the vector of excitations. The state variables $\boldsymbol{x}(t)$ are mixedphysical-domain quantities related with energy storage in the model. Examples of state variables are the displacement of the vibrating magnet, its velocity, magnetic flux as well as electrical voltages and currents of the capacitive and inductive components correspondingly. The non-state variables typically represent terminal voltages and currents which connect individual modules of the system as illustrated in Fig. 3.

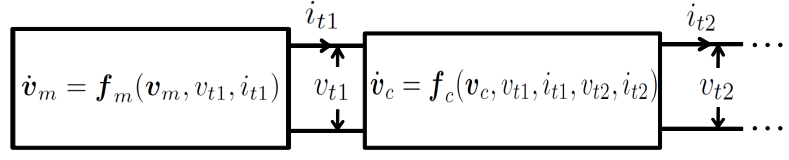

Fig. 3. Energy harvester analogue blocks showing state equations and terminal variables

For the purpose of solving Equation (1), and an automatic elimination of the non-state variables, we propose to linearise Equation (1) at each time-point $t_{n}, n=0,1, \ldots$ as shown:

$$
\left[\begin{array}{c}
\dot{\boldsymbol{x}}_{\left(t_{n}\right)} \\
\mathbf{0}
\end{array}\right]=\left[\begin{array}{ll}
\boldsymbol{J}_{x x, n} & \boldsymbol{J}_{x y, n} \\
\boldsymbol{J}_{y x, n} & \boldsymbol{J}_{y y, n}
\end{array}\right]\left[\begin{array}{l}
\boldsymbol{x}\left(t_{n}\right) \\
\boldsymbol{y}\left(t_{n}\right)
\end{array}\right]+\left[\begin{array}{c}
\boldsymbol{e}_{x}\left(t_{n}\right) \\
\mathbf{0}
\end{array}\right]
$$

where the Jacobian matrices of the linearised model at the time point $t_{n}$ are: $\boldsymbol{J}_{x x, n}=\frac{\partial \boldsymbol{f}_{x}\left(t_{n}\right)}{\partial x}, \boldsymbol{J}_{x y, n}=\frac{\partial \boldsymbol{f}_{x}\left(t_{n}\right)}{\partial y}, \boldsymbol{J}_{y x, n}=$ $\frac{\partial \boldsymbol{f}_{y}\left(t_{n}\right)}{\partial x}$ and $\boldsymbol{J}_{y y, n}=\frac{\partial \boldsymbol{f}_{y}\left(t_{n}\right)}{\partial y}$. The local linearisation error $\left(L L E_{n}\right)$ introduced at time point $t_{n}$ is:

$$
L L E_{n}=\left\|\left[\boldsymbol{x}^{*}\left(t_{n}\right)-\boldsymbol{x}\left(t_{n}\right), \boldsymbol{y}^{*}\left(t_{n}\right)-\boldsymbol{y}\left(t_{n}\right)\right]^{T}\right\|
$$

where $\boldsymbol{x}^{*}\left(t_{n}\right), \boldsymbol{y}^{*}\left(t_{n}\right)$ are the accurate values and $\boldsymbol{x}\left(t_{n}\right), \boldsymbol{y}\left(t_{n}\right)$ are the approximate values of $x(t)$ and $y(t)$ obtained due to linearisation at $t_{n}$. The $L L E$ is caused by the rejection of the Taylor expansion terms of the non-linear functions of order higher than the first. The $L L E$ can be controlled by monitoring the changes in the Jacobian elements.

At each time point $t_{n}$ the non-state variables $\boldsymbol{y}\left(t_{n}\right)$ is eliminated by solving the following linear algebraic equation, i.e the algebraic part of the linearised equation (2):

$$
\boldsymbol{J}_{y y, n} \boldsymbol{y}\left(t_{n}\right)=-\boldsymbol{J}_{y x, n} \boldsymbol{x}\left(t_{n}\right)
$$

Once the variables $\boldsymbol{y}\left(t_{n}\right)$ are calculated at $t_{n}$, the state variables $\boldsymbol{x}(t)$ are obtained at the next time point $t_{n+1}$ by applying an explicit differentiation formula, such as the Forward Euler, Adams-Bashforth or Runge-Kutta method. In the case study presented in Section III we have used the multi-step AdamsBashforth formula due to its simplicity and accuracy [9]. Hence, the solution of the linearised-state variable equation at the next time point $t_{n+1}$ is expressed as:

$$
\begin{aligned}
& \boldsymbol{x}\left(t_{n+1}\right)=\boldsymbol{x}\left(t_{n}\right) \\
& +h_{n+1} \sum_{i=0}^{p}\left[\alpha_{i}\left(\boldsymbol{J}_{x x, n} \boldsymbol{x}\left(t_{n}\right)+\boldsymbol{J}_{x y, n} \boldsymbol{y}\left(t_{n}\right)+\boldsymbol{e}_{x}\left(t_{n}\right)\right]\right.
\end{aligned}
$$


where $h_{n+1}=t_{n+1}-t_{n}$ is the current step-size and $\alpha_{i}, i=$ $0, \ldots, p$ are the coefficients of the Adams-Bashforth formula of order $p$ whose values are dependent on the varying stepsize. The local truncation error caused by the application of the $p$-th order Adams-Bashforth method is of order $O\left(h_{n+1}^{p+1}\right)$ [9]. The necessary condition for the forward march-in-time process defined by equation (5) to be numerically stable, is that the step size $h_{n+1}$ be limited. This is a well known property of explicit integration [9]. Generally, the maximum allowed step size in an iterative process of the form:

$$
\dot{\boldsymbol{x}}\left(t_{n+1}\right)=\boldsymbol{x}\left(t_{n}\right)+h_{n+1}\left(\boldsymbol{A} x\left(t_{n}\right)+\boldsymbol{b}\left(t_{n}, x\left(t_{n-1}, \ldots\right)\right)\right.
$$

where $\boldsymbol{A}$ is the point total-step matrix and $\boldsymbol{b}$ is a vector dependent on excitations and past solution points, is numerically stable if the spectral radius of the matrix $\boldsymbol{I}+h_{n+1} \boldsymbol{A}$ lies within the unity circle [10]:

$$
\rho\left(\boldsymbol{I}+h_{n+1} \boldsymbol{A}\right)<1
$$

The spectral radius is determined by the system's minimum time constant [9]. The minimum time constant is not usually known, however, the analogue parts of energy harvesters, namely the microgenerator, power conditioning circuit and supercapacitor are passive systems. Therefore the stability condition given by equation (7) can be ensured in a straightforward way by adjusting the step-size such that the point totalstep matrix is diagonally dominant [9], [10]. The proposed technique has numerous advantages. Firstly, it avoids the classical Newton-Raphson iterative process required by implicit integration which leads to long simulation CPU times (Table I) and therefore has the potential to accelerate simulations significantly. In the case study discussed in Section IV, the acceleration resulting from the application of the linearised state-space technique was two orders of magnitude, while maintaining a similar accuracy to that of a classical analogue solver. Secondly, this method of solving analogue ordinary differential equation interfaces easily with a digital kernel in a mixed-signal hardware description language. This is because the analogue solution is obtained in a single march-in-time sweep, rather than an iterative process which might involve backtracking in time. The technique is unlikely to offer a speed advantage when applied to strongly stiff systems as the stepsize must be kept small to ensure stability even if the accuracy control permits larger steps.

\section{ENERGY HARVESTER MODELS BASED ON LINEARISED STATE-SPACE FORMULATION}

To investigate the efficiency of the simulation acceleration method presented in Section II, a case study has been carried out using a practical vibration energy harvester reported in [7]. We have chosen this case study because it is complete and the most recent autonomous tunable energy harvester presented in the literature [1]. In the following subsections, we present the model equations of each component block of the tunable energy harvester (Fig. 1) and show how they can be formulated as state equations.

\section{A. Tunable microgenerator}

Fig. 4(a) shows a diagram of the electromagnetic microgenerator together with its tuning mechanism. The microgenerator is based on a cantilever structure. The coil is fixed to the base, and four magnets (which are located on both sides of the coil) form the proof mass. The tuning mechanism uses magnetic force to change the effective stiffness of the cantilever which leads to a change of resonant frequency. One tuning magnet is attached to the end of the cantilever beam and the other tuning magnet is connected to a linear actuator. The linear actuator is controlled by a microcontroller and moves the magnet to desired position so that the resonant frequency of the microgenerator always matches the frequency of the ambient vibration. Fig. 4(b) shows a photo of the microgenerator which is used to validate the proposed technique.

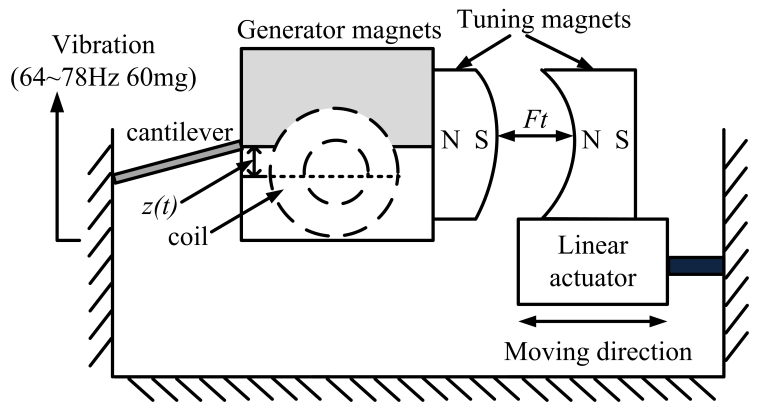

(a) Mechanical part

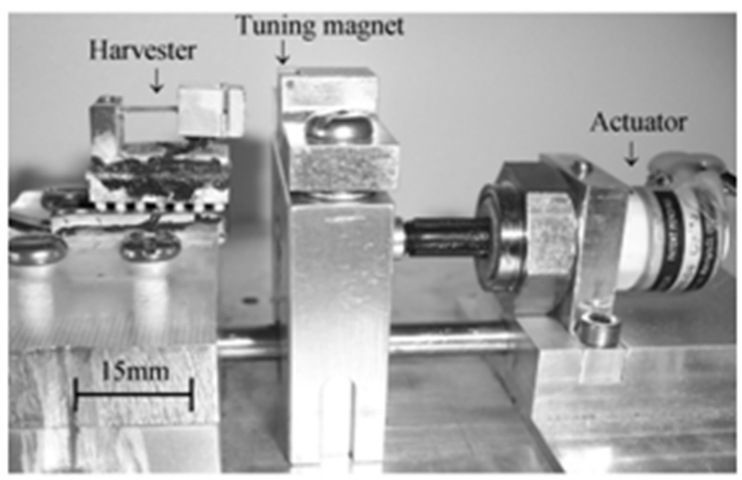

(b) Photo of tunable microgenerator [7]

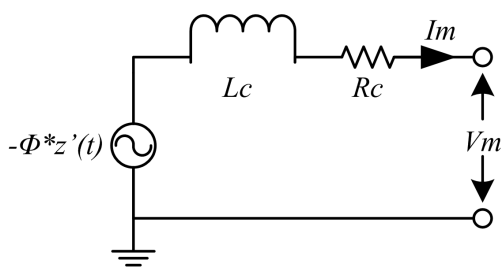

(c) Electrical part

Fig. 4. Tunable electromagnetic microgenerator [7]

The dynamic model of the microgenerator is [2]:

$$
m \frac{\mathrm{d}^{2} z(t)}{\mathrm{d} t^{2}}+c_{p} \frac{\mathrm{d} z(t)}{\mathrm{d} t}+k_{s} z(t)+F_{e m}+F_{t_{-} z}=F_{a}
$$

where $m$ is the proof mass, $z(t)$ is the relative displacement between the mass and the base, $c_{p}$ is the parasitic damping 
factor, $k_{s}$ is the effective spring stiffness, $F_{e m}$ is the electromagnetic force, $F_{t_{-} z}$ is the $z$ component of tuning force $F_{t}$ and $F_{a}$ is the input acceleration force.

Fig. 4(c) is the equivalent circuit of the microgenerator. The electromagnetic voltage generated in the coil is:

$$
V_{e m}=-\Phi \frac{\mathrm{d} z(t)}{\mathrm{d} t}
$$

where $\Phi=N B l$ is the magnetic flux through the coil and $N$ is the number of coil turns, $B$ is the magnetic field and $l$ is the effective length. The output voltage is:

$$
V_{m}(t)=V_{e m}-R_{c} i_{L}(t)-L_{c} \frac{\mathrm{d} i_{L}(t)}{\mathrm{d} t}
$$

where $R_{c}$ and $L_{c}$ are the resistance and inductance of the coil respectively and $i_{L}(t)$ is the current through the coil. The electromagnetic force is calculated as:

$$
F_{e m}=\Phi i_{L}(t)
$$

The resonant frequency of the tuned microgenerator $\left(f_{r}^{\prime}\right)$ is:

$$
f_{r}^{\prime}=f_{r} \sqrt{1+\frac{F_{t}}{F_{b}}}
$$

where $f_{r}$ is the un-tuned resonant frequency, $F_{t}$ is the tuning force between two magnets and $F_{b}$ is the buckling load of a cantilever. In the microgenerator model, the state variables are the relative velocity and displacement between the coil and magnets $\left(\frac{d z(t)}{d t}\right.$ and $\left.z(t)\right)$, plus the inductor current $\left(i_{L}(t)\right)$. The terminal variables are $V_{m}$ and $I_{m}$ (obviously $I_{L}(t)=I_{m}$ ). The above equations 8 to 11 can be rearranged and written in state-space form:

$$
\begin{aligned}
\frac{\mathrm{d}}{\mathrm{d} t}\left[\begin{array}{c}
z(t) \\
\frac{\mathrm{d} z(t)}{\mathrm{d} t} \\
i_{L}(t)
\end{array}\right] & =\left[\begin{array}{ccc}
1 & 0 & 0 \\
\frac{-c_{p}}{m} & \frac{-k_{s}}{m} & \frac{-\Phi}{m} \\
\frac{-\Phi}{L_{c}} & 0 & \frac{-R_{c}}{L_{c}}
\end{array}\right]\left[\begin{array}{c}
z(t) \\
\frac{\mathrm{d} z(t)}{\mathrm{d} t} \\
i_{L}(t)
\end{array}\right] \\
& +\left[\begin{array}{cc}
0 & 0 \\
0 & 0 \\
\frac{-1}{L_{c}} & 0
\end{array}\right]\left[\begin{array}{c}
V_{m} \\
I_{m}
\end{array}\right]+\left[\begin{array}{c}
0 \\
\frac{F_{a}-F_{t z}}{m} \\
0
\end{array}\right]
\end{aligned}
$$

\section{B. Power processing}

A 5-stage Dickson voltage multiplier has been used to rectify and boost up the generated AC voltage (Fig. 5(a)). In this case because the circuit contains non-linear components (diodes), it is necessary to linearise the model to produce state-space equations. The Shockley diode equation is: $I_{d}=$ $I_{s}\left(e^{V_{d} / V_{t}}-1\right)$ where $V_{d}$ and $I_{d}$ are the diode voltage and current, $I_{s}$ is the saturation current and $V_{t}$ is the thermal voltage. The linearised diode equation is: $I_{d}=G V_{d}+J$ where $G$ and $J$ are the values of conductor and ideal current source. According to the Shockley diode equation, $G$ and $J$ can be written as piecewise-linear functions of $V_{d}$. The values of $G$ and $J$ are stored in a look-up table for different values of $V_{d}$. Since explicit integration is used to solve the state equations, the model is linear at each time step. Fig. 5(b) shows the linearised voltage multiplier model. The state variables of the power processing circuit model are the voltages across each capacitor $\left(V_{1}\right.$ to $\left.V_{5}\right) . C_{i}, G_{i}$ and $J_{i}(i=1$ to 5) are the capacitor, conductor and current values of the linearised model. The terminal variables are $V_{m}, I_{m}, V_{c}$ and $I_{c}$ (obviously $V_{c}=V_{5}$ ). The state equations are:

$$
\begin{aligned}
\frac{\mathrm{d}}{\mathrm{d} t}\left[\begin{array}{l}
V_{1} \\
V_{2} \\
V_{3} \\
V_{4} \\
V_{5}
\end{array}\right]= & {\left[\begin{array}{ccccc}
\frac{-G_{1}-G_{2}}{C_{1}} & \frac{-G_{2}}{C_{1}} & 0 & 0 & 0 \\
\frac{-G_{2}}{C_{2}} & \frac{-G_{2}-G_{3}}{C_{2}} & \frac{-G_{3}}{C_{2}} & 0 & 0 \\
0 & \frac{-G_{3}}{C_{3}} & \frac{-G_{3}-G_{4}}{C_{3}} & \frac{-G_{4}}{C_{3}} & 0 \\
0 & 0 & \frac{-G_{4}}{C_{4}} & \frac{-G_{4}-G_{5}}{C_{4}} & \frac{-G_{5}}{C_{4}} \\
0 & 0 & 0 & \frac{-G_{5}}{C_{5}} & \frac{-G_{5}}{C_{5}}
\end{array}\right]\left[\begin{array}{l}
V_{1} \\
V_{2} \\
V_{3} \\
V_{4} \\
V_{5}
\end{array}\right] } \\
& +\left[\begin{array}{cccc}
\frac{G_{1}+G_{2}}{C_{1}} & 0 & 0 & 0 \\
\frac{G_{2}+G_{3}}{C_{2}} & 0 & 0 & 0 \\
\frac{G_{3}+G_{4}}{C_{3}} & 0 & 0 & 0 \\
\frac{G_{4}+G_{5}}{C_{4}} & 0 & 0 & 0 \\
\frac{G_{5}}{C_{5}} & 0 & 0 & \frac{-1}{C_{5}}
\end{array}\right]\left[\begin{array}{c}
V_{m} \\
I_{m} \\
V_{c} \\
I_{c}
\end{array}\right]+\left[\begin{array}{c}
\frac{J_{1}-J_{2}}{C_{1}} \\
\frac{J_{3}-J_{2}}{C_{2}} \\
\frac{J_{3}-J_{4}}{C_{3}} \\
\frac{J_{5}-J_{4}}{C_{4}} \\
\frac{J_{5}}{C_{5}}
\end{array}\right]
\end{aligned}
$$

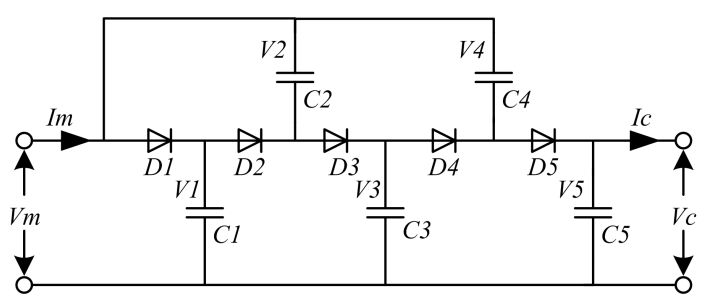

(a) 5-stage Dickson voltage multiplier

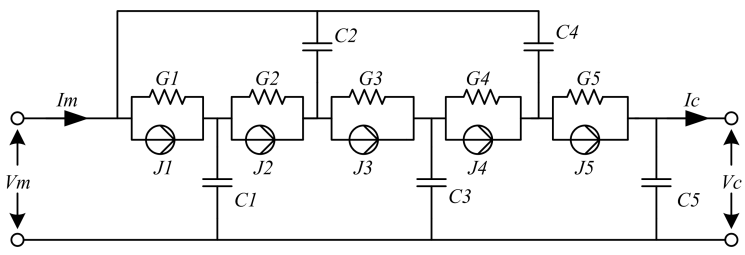

(b) Linearised voltage multiplier model

Fig. 5. Voltage multiplier as power processing circuit

The piece-wise linear tabular models are an additional measure to save computation time. Due to the forward march-intime nature of the explicit integration algorithm, the required Jacobian values can be retrieved from the look-up tables fast, without the need to evaluate complex, physical equations. To maintain high modelling accuracy the granularity of the piecewise linear models can be arbitrarily fine since the size of the look-up tables does not affect the simulation speed.

\section{Supercapacitor and equivalent load resistor}

The supercapacitor model has been chosen for its good accuracy [11]. The model (Figure 6) is comprised of a network of three RC circuits which model the charge redistribution process within the supercapacitor. The state variables of the supercapacitor model are the voltages across each capacitor $\left(V_{i}, V_{d}\right.$ and $\left.V_{l}\right)$. The terminal variables are $V_{c}$ and $I_{c}$. The state equations are:

$$
\begin{array}{r}
\mathrm{d}\left[\begin{array}{l}
V_{i} \\
V_{d} \\
V_{l}
\end{array}\right]=\left[\begin{array}{ccc}
\frac{-1}{R_{i}\left(C_{i 0}+C_{i 1}\right)} & 0 & 0 \\
0 & \frac{-1}{R_{d} C_{d}} & 0 \\
0 & 0 & \frac{-1}{R_{l} C_{l}}
\end{array}\right]\left[\begin{array}{c}
V_{i} \\
V_{d} \\
V_{l}
\end{array}\right] \\
+\left[\begin{array}{cc}
\frac{1}{R_{i}\left(C_{i 0}+C_{i 1}\right)} & 0 \\
\frac{1}{R_{d} C_{d}} & 0 \\
\frac{1}{R_{l} C_{l}} & 0
\end{array}\right]\left[\begin{array}{c}
V_{c} \\
I_{c}
\end{array}\right]
\end{array}
$$




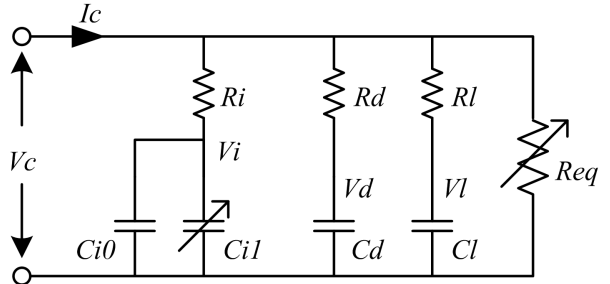

Fig. 6. Supercapacitor and equivalent load resistor model

$R_{e q}$ (Figure 6) is the equivalent load resistor representing the power consumption of the actuator and the microcontroller. The $R_{e q}$ values for different operation modes of the microcontroller are:

$$
R_{e q}= \begin{cases}1.0 e 9 \Omega \text { when microcontroller is in sleep mode } \\ 33 \Omega & \text { when microcontroller wakes up } \\ 16.7 \Omega & \text { when actuator performs tuning }\end{cases}
$$

\section{Microcontroller}

In order for a tunable energy harvester (Fig. 1) to work autonomously, both the actuator and the microcontroller need to be powered by the energy storage. The time required for the harvester to generate enough energy to perform one frequency tuning process determines the harvester's duty cycle. This tuning process is controlled by a microcontroller. Since the microcontroller is purely digital, there are no state equations needed to model the microcontroller. The flow chart of the microcontroller tuning process is shown in Fig. 7. Standard SystemC modules were used to model the digital control process. As can be seen in Fig. 7, a watchdog timer wakes the microcontroller periodically and the microcontroller first detects if there is enough energy stored in the supercapacitor. If there is not enough energy, the microcontroller goes to sleep and waits for the watchdog timer again. If there is enough energy, the microcontroller will then detect the ambient vibration frequency to see if it matches the microgenerator's resonant frequency. If there is a difference between the vibration frequency and the resonant frequency, the microcontroller will start the tuning process by controlling the actuator to move the tuning magnet to the desired position (Fig. 4(a)).

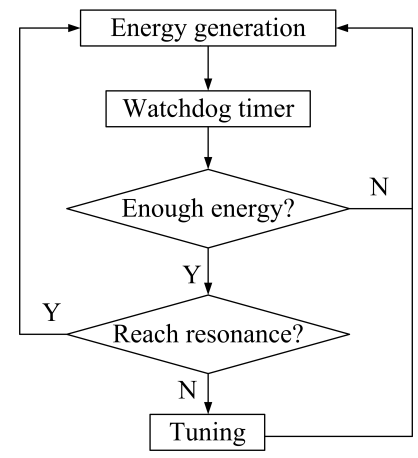

Fig. 7. Flow chart of the microcontroller digital process

\section{E. Complete mixed-technology energy harvester model}

When combining the three component blocks (microgenerator, power processing and supercapacitor) together, the terminal variables of each component block will be represented by state variables and eliminated. This enables the whole energy harvester model to be described by state equations and these state equations can be solved using explicit integration method, such as Adams-Bashforth. The combination of mixedtechnology energy harvester model is automated by the method described in Section II. Applying Equation 4 to matrices 13, 14 and 15 and combining the three matrices will generate a complete set of state equations of the complete model. The complete state-space matrix is omitted here due to space limitations. To give an insight into the model size, the statespace model of a complete energy harvester consists of a 11 by 11 matrix of state equations.

\section{Simulation Results}

A SystemC-A model of the tunable energy harvester [7] has been developed using the proposed linearised state-space technique and simulated. For comparison, a VHDL-AMS model of the same tunable energy harvester has been developed and simulated with SystemVision from Mentor Graphics using the built-in Newton-Raphson method. Two test scenarios have been carried out. Scenario 1 is based on narrow tuning range; varying the frequency by $1 \mathrm{~Hz}$ and Scenario 2 is based on wide tuning range; varying the frequency of $14 \mathrm{~Hz}$ which is the maximum tuning range of the design [7]. Because the VHDL-AMS and SystemC-A models are based on the same equations and their simulation results are almost identical, only simulation waveforms generated by the SystemC-A model are compared with experimental measurements of the practical tunable energy harvester.

Fig. 8(a) shows the power output from the microgenerator during the $1 \mathrm{~Hz}$ tuning process. The waveform shows that when the ambient frequency shifts from 70 to $71 \mathrm{~Hz}$, as expected the output power drops down and goes up before and after tuning. The simulated RMS power is $118 \mu \mathrm{W}$ when the microgenerator is tuned at $70 \mathrm{~Hz}$ and $117 \mu \mathrm{W}$ when it is tuned at $71 \mathrm{~Hz}$. These values match well with the reported practical test value of $116 \mu \mathrm{W}$ as presented in [2]. Fig. 8(b) shows the comparison between simulated and experimental measured voltage waveforms across the supercapacitor of the energy harvester. As can be seen, the simulation waveform correlates well with the experimental measurement. In Scenario 2, we increase the frequency variation to $14 \mathrm{~Hz}$ which presents a more challenging simulation case due to the wider frequency range. Yet there is close correlation between simulation and experimental waveforms as can be seen in Fig. 9. This provides evidence that our technique is accurate even for energy harvester with a wide frequency tuning range. Whilst Fig. 8(b) and 9 show close agreements between the simulation and experimental results, there are some differences between the waveforms. This is because the HDL model of the energy harvester cannot incorporate every aspect of a practical system, such as leakage and parasitic loss.

Table II shows CPU times of both the proposed state-space simulation and the Mentor Graphics' SystemVision simulation for the energy harvester in Scenario 1 and 2. It can be seen that 
the state-space technique can accelerate the simulation by two orders of magnitude compared to that of the commerciallyavailable simulator.

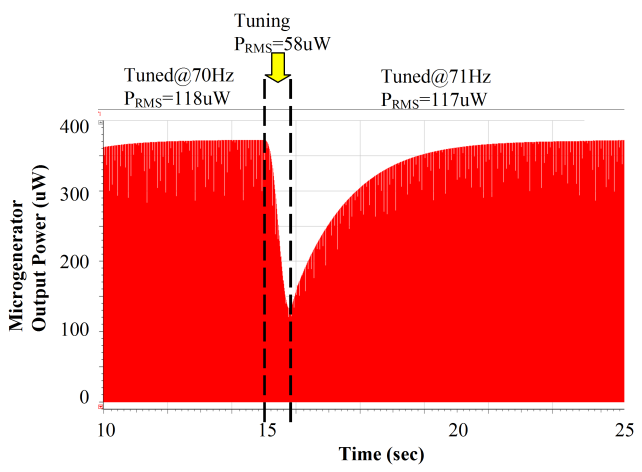

(a) Simulation result of output power from microgenerator

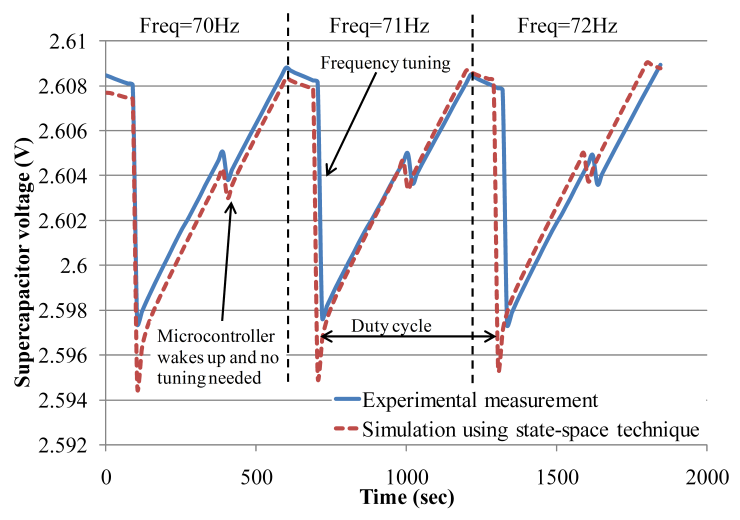

(b) Simulation and experimental results of supercapacitor voltage

Fig. 8. Scenario 1: Simulation and experimental results of $1 \mathrm{~Hz}$ tuning

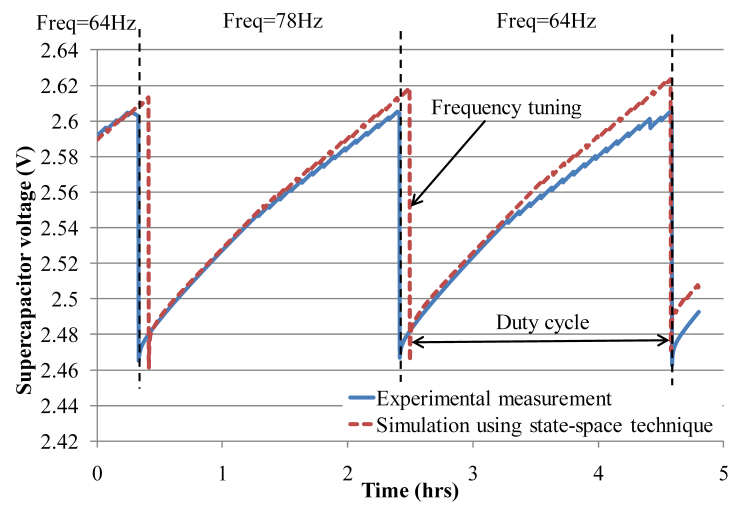

Fig. 9. Scenario 2: Simulation and experimental results of $14 \mathrm{~Hz}$ tuning

TABLE II

CPU TIMES OF EXISTING AND PROPOSED SIMULATION TECHNIQUES

\begin{tabular}{|c|c|c|}
\hline & Existing technique & Proposed technique \\
\hline Simulator & SystemVision & MS Visual C++ \\
\hline HDL & VHDL-AMS & SystemC-A \\
\hline Integration method & Newton-Raphson & Adams-Bashforth \\
\hline CPU time for Scenario 1 & $2185 \mathrm{sec}$ & $20.3 \mathrm{sec}$ \\
\hline CPU time for Scenario 2 & 7 hours & $228 \mathrm{sec}$ \\
\hline
\end{tabular}

\section{CONCLUSiON}

This work is the first investigation into accelerating the simulation time of energy harvesting systems, an important type of systems employed in emerging applications. The main motivation for the research into fast simulation of energy harvesters is development of an automated design approach by which the best topology and optimal parameters of energy harvester are obtained iteratively using multiple simulations. The proposed linearised state-space technique provides a vital step in achieving this goal. Our experimental and analytical studies show that explicit integration of the linearised statespace equations can significantly decrease the simulation time of such systems whilst maintaining high simulation accuracy as demonstrated by excellent correlation between experimental and simulation results. While we demonstrated the effectiveness of our approach using an electromagnetic microgenerator, this is a generic approach which can be applied to other types of microgenerators such as electrostatic or piezoelectric. All that is required are the model equations of each component block of the tunable energy harvester as in Section III.

\section{ACKNOWLEDGMENT}

This work was supported in part by the Engineering and Physical Sciences Research Council (EPSRC), UK, under grant number EP/G067740/1 "Next Generation Energy-Harvesting Electronics: Holistic Approach," website: www.holistic.ecs.soton.ac.uk

\section{REFERENCES}

[1] D. Zhu, J. Tudor, and S. Beeby, "Strategies for increasing the operating frequency range of vibration energy harvesters: a review," Measurement Science and Technology, vol. 21, no. 2, p. 022001, 2010.

[2] D. Zhu, S. Roberts, J. Tudor, and S. Beeby, "Design and experimental characterization of a tunable vibration-based electromagnetic microgenerator," Sensors and Actuators A: Physical, vol. 158, no. 2, pp. 284293, 2010.

[3] D. Hohlfeld, S. Matova, and R. van Schaijk, "System-level modeling and simulation of a frequency-tunable electrostatic energy harvester," in Thermal, Mechanical and Multi-Physics simulation and Experiments in Microelectronics and Microsystems, 2009. EuroSimE 2009. 10th International Conference on, 26-29 April 2009, pp. 1-7.

[4] K. Rao, P. Mitcheson, and T. Green, "Imperial college energy-harvesting simulator (ICES)," http://www3.imperial.ac.uk/controlandpower/ research/portfoliopartnership/projects/powermems/simulationtoolkit/, February 17, 2010.

[5] W.-J. Wu, Y.-F. Chen, Y.-Y. Chen, C.-S. Wang, and Y.-H. Chen, "Smart wireless sensor network powered by random ambient vibrations," in Systems, Man and Cybernetics, 2006. SMC 'O6. IEEE International Conference on, 8-11 Oct. 2006, pp. 2701-2708.

[6] L. Wang, T. Kazmierski, B. Al-Hashimi, S. Beeby, and D. Zhu, "An automated design flow for vibration-based energy harvester systems," in Design, Test and Automation in Europe (DATE 2009), April 20-24, 2009, pp. 1391-1396.

[7] I. Ayala-Garcia, D. Zhu, J. Tudor, and S. Beeby, "Autonomous tunable energy harvester," in PowerMEMS 2009, 1-4 December 2009, pp. 49-52.

[8] H. Al-Junaid, SystemC-A: Analogue and Mixed-Signal Language For High Level System Design. PhD Thesis, University of Southampton, 2006.

[9] L. O. Chua and P. Y. Lin, Computer-Aided Analysis of Electronic Circuits: Algorithms and Computational Techniques. Prentice Hall Professional Technical Reference, 1975.

[10] R. Varga, Matrix Iterative Analysis. Springer Series in Computational Mathematics, 2000.

[11] L. Zubieta and R. Bonert, "Characterization of double-layer capacitors for power electronics applications," Industry Applications, IEEE Transactions on, vol. 36, pp. 199-205, 2000. 